\title{
Application of FLEET Velocimetry in the NASA Langley 0.3-meter Transonic Cryogenic Tunnel
}

\author{
Ross A. Burns ${ }^{1}$ and Paul M. Danehy ${ }^{2}$ \\ NASA Langley Research Center, Hampton, Virginia 23681 \\ and \\ Benjamin R. Halls ${ }^{3}$ and Naibo Jiang ${ }^{4}$ \\ Spectral Energies, LLC, Dayton, Ohio 45431
}

\begin{abstract}
Femtosecond laser electronic excitation and tagging (FLEET) velocimetry is demonstrated in a large-scale transonic cryogenic wind tunnel. Test conditions include total pressures, total temperatures, and Mach numbers ranging from 15 to 58 psia, 200 to $295 \mathrm{~K}$, and 0.2 to 0.75 , respectively. Freestream velocity measurements exhibit accuracies within 1 percent and precisions better than $1 \mathrm{~m} / \mathrm{s}$. The measured velocities adhere closely to isentropic flow theory over the domain of temperatures and pressures that were tested. Additional velocity measurements are made within the tunnel boundary layer; virtual trajectories traced out by the FLEET signal are indicative of the characteristic turbulent behavior in this region of the flow, where the unsteadiness increases demonstrably as the wall is approached. Mean velocities taken within the boundary layer are in agreement with theoretical velocity profiles, though the fluctuating velocities exhibit a greater deviation from theoretical predictions.
\end{abstract}

\section{Nomenclature}

Symbols

$a$
$f$
$I$
$K$
$n$
$N$
$p$
$P$
$s$
$t$
$T$
$u, v$
$x$
$y$

$=$ acceleration $\left[\mathrm{m} / \mathrm{s}^{2}\right]$

$=$ frequency $[\mathrm{Hz}]$

$=$ signal intensity [a.u.]

$=$ number of samples in a data set

$=$ number of points considered in a fit

$=$ total number of points in a given burst

$=$ number of degrees of freedom in a fit

$=$ pressure [psia]

$=$ distribution width fit coefficient $\left[\mathrm{px}^{2}\right]$

$=$ time $[\mathrm{s}]$

$=$ temperature $[\mathrm{K}]$

$=$ velocity $[\mathrm{m} / \mathrm{s}]$

$=$ stream-wise coordinate direction

$=$ wall-normal coordinate direction

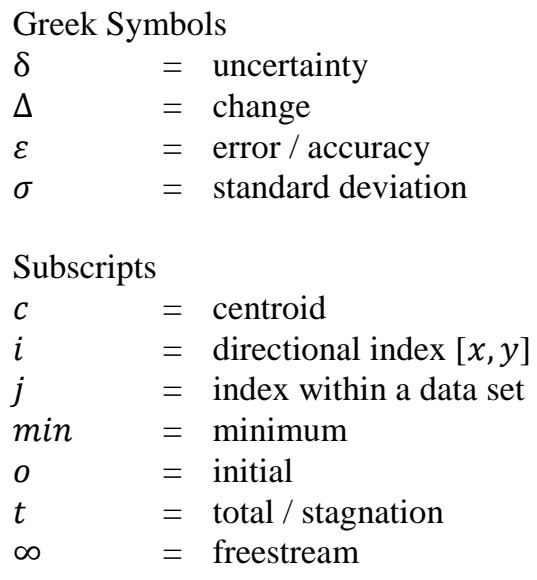

\section{Introduction}

$\mathrm{T}$

RANSONIC wind tunnels have been important research tools for over three-quarters of a century. Their ability to accurately recreate a wide range of flight Mach numbers for both commercial and military propulsion and

\footnotetext{
${ }^{1}$ Research Scholar, National Institute of Aerospace, Hampton, VA, AIAA Member

${ }^{2}$ Research Scientist, Advanced Measurements and Data Systems Branch, AIAA Associate Fellow

${ }^{3}$ Research Scientist, Spectral Energies, LLC, AIAA Student Member

${ }^{4}$ Research Scientist, Spectral Energies, LLC, AIAA Senior Member
}

1

American Institute of Aeronautics and Astronautics 
transit applications has proven invaluable in advancing the state-of-the-art in these technological areas. More recently, the development and refinement of large-scale cryogenic transonic wind tunnel facilities has further advanced the ground-testing capabilities available to researchers. ${ }^{1}$ By utilizing very low temperature gas as the working fluid, extremely high Reynolds numbers are attainable through the modulation of both the gaseous density and viscosity. ${ }^{2,3,4}$ Known collectively as transonic cryogenic tunnels (TCTs), operating unit Reynolds numbers in these facilities exceeding $3 \times 10^{8}$ per meter have been reported. ${ }^{3}$ Another important consequence of this operational principle is that the dynamic pressures acting on test articles within the facility are not dramatically affected by changes in the Reynolds number. Ground testing at full-scale Reynolds numbers is made possible with this unique combination of features. ${ }^{3,5}$

While transonic cryogenic tunnels can reproduce flight Reynolds numbers, performing optical flow measurements within them has proven challenging historically. The reasons for this are several. First, these facilities generally operate at very high pressures, necessitating a rugged construction that often limits or eliminates optical access to the flowfield. Second, the close proximity of the cryogenic environment of the flowfield to the ambient environments can cause a range of problems including condensation and freezing of water vapor onto optical surfaces and large density fluctuations both within and around the facilities. The former of these issues can cause the facilities to become opaque to optical measurement techniques, and the latter has been known to cause significant beam-steering and wave-front distortion effects. ${ }^{3,4}$ Finally, the large mechanical drives used in these facilities are known to cause vibrations over a broad range of frequencies in any adjacent or attached equipment. These vibrations are problematic when applying any technique for which optical alignment is crucial, and are known to interfere with various optical diagnostic techniques applied in these tunnels. ${ }^{5,6}$ Finally, the introduction of particles or toxic gases, which is common practice in various optical measurement techniques, can be difficult or prohibitively hazardous in TCT facilities. As a consequence of these physical constraints placed on any measurement technique applied in these facilities, a very limited number of optical flowfield measurements techniques have been successfully applied in cryogenic tunnels. ${ }^{6,7}$

Enabling the use of laser-based and other optical diagnostic techniques for measuring flow quantities such as velocity and surface pressure is a crucial step towards enhancing the overall measurement capabilities in TCT facilities. Although these techniques have found broad appeal in the aerospace community because of their inherently non-intrusive nature (and thus the valuable data they can provide), the application of optical diagnostics in transonic cryogenic tunnels has been limited. Temperature sensitive paint (TSP) has been successfully implemented in a number of TCT facilities. ${ }^{8,9,10,11}$ While traditional TSP formulations lose their temperature sensitivity at cryogenic temperatures, new formulations were developed to operate in the cryogenic facilities, having effective temperature ranges from $90 \mathrm{~K}$ to $300 \mathrm{~K}$, though there is some variability depending on the formulation. ${ }^{11}$, ${ }^{12}$ This so-called cryoTSP technique has been used rather extensively for studying laminar to turbulent transition on airfoils in several TCT facilities including the European Transonic Windtunnel (ETW) and its pilot facility, Japan's NAL 0.1-m Transonic Cryogenic Wind Tunnel, and the transonic facilities of the German-Dutch Windtunnels (DNW-KKK and DNW-KRG). ${ }^{10},{ }^{11}$ Pressure sensitive paint (PSP) has also found success in its application to cryogenic facilities. A number of different researchers have been able to implement pressure-sensitive paint (PSP) for measuring the surface pressure on different types of models including a delta-wing airfoil at Japan's NAL 0.1-m Transonic Cryogenic Wind Tunnel ${ }^{\mathbf{1 3}}$, a super-critical airfoil in NASA Langley's $0.3-\mathrm{m}$ Transonic Cryogenic Tunnel (0.3-m TCT) ${ }^{\mathbf{1 4}}$, and a blended-wing body ${ }^{\mathbf{1 5}}$ and sub-scale commercial transport in NASA Langley's National Transonic Facility (NTF) ${ }^{\mathbf{1 6}}$.

Measurements involving schlieren and other techniques sensitive to density have been implemented in transonic cryogenic facilities as well. Notably, standard schlieren ${ }^{3},{ }^{4}$, focusing schlieren ${ }^{17}$, and shadowgraphy have been implemented in the $0.3-\mathrm{m}$ TCT facility with moderate success. It was in the implementation of shadowgraphy that the beam-steering and wave-front-distortion effects were first recognized in these types of facilities. One study in Langley's 0.3-m TCT attempted to utilize Rayleigh scattering as a measure of the freestream density. ${ }^{18}$ While the application was successful as a demonstration, many experimental difficulties were encountered such as a large unsteadiness in their probe laser energy caused by the temperature variations in the facility and potentially the condensation of carbon dioxide found in the $\mathrm{LN}_{2}$ injected in the facility.

The application of velocity-imaging diagnostics has been limited largely to the European transonic wind tunnel facilities. Applications of Doppler global velocimetry (DGV) and particle image velocimetry (PIV) have been reported in the literature. ${ }^{10}, 11$ The implementation of both of these techniques faced considerable engineering challenges. Most notably, the need to seed particles into the flow and the limited optical access posed significant challenges. ${ }^{19}$ To handle the seeding of particles, warm nitrogen saturated with water vapor was injected into the facility to allow for nucleated ice crystals to form, which acted as the light-scattering medium if the test conditions were conducive. Elaborate electro-mechanical systems were required to adjust the camera viewing angles and light 
sheets as necessary. PIV has been utilized regularly in both the ETW and DNW-KKK facilities, studying phenomena such as wake flow from a high-lift airfoil ${ }^{20}, 21$ and nacelle vortex propagation ${ }^{22}$ with two- and threecomponent velocity measurements. DGV has had a more limited number of applications due to both a more stringent requirement on the seeding quality and lower signal-to-noise ratios attained in the image acquisition. Experiments looking at an airfoil wake and wing-tip vortex have been conducted in ETW with modest success using DGV, though these tests were preliminary. ${ }^{23}$

At Langley Research Center, the measurement of velocity in TCT facilities have traditionally been limited to inferred velocities from pressure measurements. Only two applications of laser-based velocity measurements have been reported in these facilities, both of which were conducted in the $0.3-\mathrm{m}$ TCT. One study utilized Laser Doppler Velocimetry (LDV) to make measurements of both the freestream velocity and the velocity at a point over an airfoil model. ${ }^{5,7}$ The second study utilized Laser Transit Anemometry (LTA) to make measurements of the velocity in the vicinity of a circular cylinder and flow angularity. ${ }^{6,7}$ In the first of these studies, a naturally occurring scattering medium was utilized to make the measurement, which was hypothesized to be droplets of $\mathrm{LN}_{2}$ that had not completely evaporated after injection into the facility. The study utilizing LDV found remarkable accuracy in their measured velocities with respect to calculated velocities within the tunnel, and minimal uncertainties associated with their measurement system (though the measured facility vibrations induced some error). An unfortunate aspect of this study was that the scattering medium required the tunnel to be operated at its maximum conditions (in terms of Reynolds number) to achieve sufficient seeding densities. Furthermore, at elevated temperatures, no reliable measurements could be made, since the seeding density was largely a function of the amount of $\mathrm{LN}_{2}$ being injected. ${ }^{5,7}$ The second of these studies utilized nucleated ice crystals issuing from a jet of shop air from the facility, whose residual humidity condensed and crystalized when exposed to the cold freestream conditions. Though reasonable results were obtained, it was unclear whether there was sufficient natural seeding density present to make these measurements accurately. ${ }^{6,7}$ As an additional consideration, neither of the aforementioned approaches to particle seeding would function above freezing conditions, which are encountered when air or warm nitrogen testing is sometimes done in both $0.3-\mathrm{m}$ TCT and NTF.

These previous attempts at velocimetry, though of high quality in terms of the assessments being performed, were limited in utility. These limitations stemmed, in part, from their dependence on particulates and the conditions within the flow facility. Furthermore, the measurements being made were inherently one-dimensional and had a rather complicated optical setup including crossed laser beams, which made them more susceptible to errors caused by the facility vibrations. Herein a relatively new technique is explored for performing velocimetry within the same confines as these previous experiments, femtosecond laser electronic excitation and tagging, or FLEET for brevity. ${ }^{24}$ FLEET belongs to a class of techniques known as molecular tagging velocimetry (MTV), in which specific atoms or molecules are 'tagged' by exciting fluorescence with an appropriately tuned laser. By imaging the spatial evolution of this fluorescence signal at a known time delay, a multi-dimensional velocity can be inferred. There are a variety of MTV techniques, which utilize different molecules including NO-tagging velocimetry ${ }^{25}$, VENOM (vibrationally-excited nitric oxide monitoring) ${ }^{26}$, HTV (hydroxyl-tagging velocimetry) ${ }^{27},{ }^{28}$, APART (air photolysis and recombination tracking) ${ }^{29}$, and RELIEF (Raman Excitation + Laser Induced Electronic Fluorescence) ${ }^{30}$ among many others. Optical setups used in these techniques can become complex and may require delicate alignment that make them susceptible to errors from the facility vibrations. Many of these techniques also require specific (sometimes toxic) gases to be seeded directly into the flow or for oxygen to be present for them to function properly, making them impractical to apply in transonic cryogenic facilities which usually operate on pure $N_{2}$. Though subject to its own limitations (i.e., can act as a thermal perturbation to the flow), FLEET stands apart from these other techniques in a number of beneficial ways. First, the excited molecule is naturally occurring nitrogen, which is present in abundance in both air- and $\mathrm{N}_{2}$ - based facilities. Second, the excitation of fluorescence is achieved in a single step in the near-infrared portion of the spectrum using a single laser. The optical system is generally less complex, and very high transmittances are afforded by the silica glasses commonly used in maintaining the pressure shell within these facilities. Third, the lifetime of the fluorescence is very long lived, which allows both high measurement precision and measurement of low velocities, further conducive to use in the transonic facilities. By contrast, NO MTV has a measurement uncertainty on the order of $30 \mathrm{~m} / \mathrm{s}$ and thus is suited only to measure hypersonic flows. ${ }^{31}$ Finally, the FLEET signal intensity has previously been shown to increase with high pressures ${ }^{32}$, and we observed in laboratory experiments (see Fig. 1 below) that the signal increases at low temperatures, which suit TCT environments well. This unique combination of features makes FLEET an ideal candidate for velocimetry within the cryogenic environments described previously.

This paper documents the first application of FLEET velocimetry in a transonic cryogenic flow facility. Accuracy, precision, and uncertainty of freestream velocity measurements are assessed. The velocities measured in the tunnel freestream and boundary layer are then analyzed in the context of the flow physics. The paper is 
structured as such: following this introduction, a brief synopsis of FLEET spectral theory is given. These sections are succeeded by a description of the experimental program including a description of the flow facility and the optical systems used in the collection of the data. Subsequently, the results and discussion thereof are presented followed by the conclusions.

\section{FLEET Spectral Theory}

FLEET signal generation is the consequence of an intense, multi-photon absorption process involving molecular nitrogen $\left(N_{2}\right)$. A femtosecond laser pulse, with a central wavelength generally near $800 \mathrm{~nm}$ is brought to a focus. In the process, some fraction of the molecular nitrogen present near the focal region absorbs several photons. The nitrogen can become rovibronically excited $\left(N_{2}\left(X^{1} \Sigma_{g}^{+}\right) \rightarrow N_{2}\left(C^{3} \Pi_{u}\right)\right)$, dissociate and then recombine $\left(N_{2}\left(X^{1} \Sigma_{g}^{+}\right) \rightarrow\right.$ $N_{2}\left(B^{3} \Pi_{g}\right)$ ), or ionize $\left(N_{2}\left(X^{1} \Sigma_{g}^{+}\right) \rightarrow N_{2}^{+}\left(B^{2} \Sigma_{u}^{+}\right)\right)$in this fashion; the FLEET signal arises from the de-excitation of these different states.

Emission spectra observed from nitrogen excited by intense femtosecond pulses show a complicated structure (refer to Fig.1). There are two general components to the FLEET emission spectrum attributed to the different deexcitation pathways. First, there is a series of discrete emission bands found in the near-UV portion of the spectrum $(\lambda<400 \mathrm{~nm})$. These peaks are theorized to come from the combined decay of the first negative system of $N_{2}^{+}$ $\left(N_{2}^{+}\left(B^{2} \Sigma_{u}^{+}\right) \rightarrow N_{2}^{+}\left(X^{2} \Sigma_{g}^{+}\right)\right)$and the second positive emissions of $N_{2}\left(N_{2}\left(C^{3} \Pi_{u}\right) \rightarrow N_{2}\left(B^{3} \Pi_{g}\right)\right)$. The decay lifetimes associated with this component are relatively short - generally less than $1 \mu \mathrm{s} .{ }^{33}$ The remaining FLEET signal is mostly contained in the visible portion of the spectrum $(500-800 \mathrm{~nm})$, arising from the first positive system $\left(N_{2}\left(B^{3} \Pi_{g}\right) \rightarrow N_{2}\left(A^{3} \Sigma_{u}^{+}\right)\right)$. The recombination process, which feeds the aforementioned nitrogen B state, is considered to be rate-limiting for the overall decay of the FLEET signal, leading to long-lived emission with characteristic lifetimes of order tens to hundreds of $\mu \mathrm{s} .{ }^{34}$ Velocimetry derived the FLEET signal typically utilizes the visible portion of the FLEET emission due to the longer lifetime. Refer to Refs. 33 and 34 for a more detailed discussion of the photophysical properties of FLEET excitation and emission.

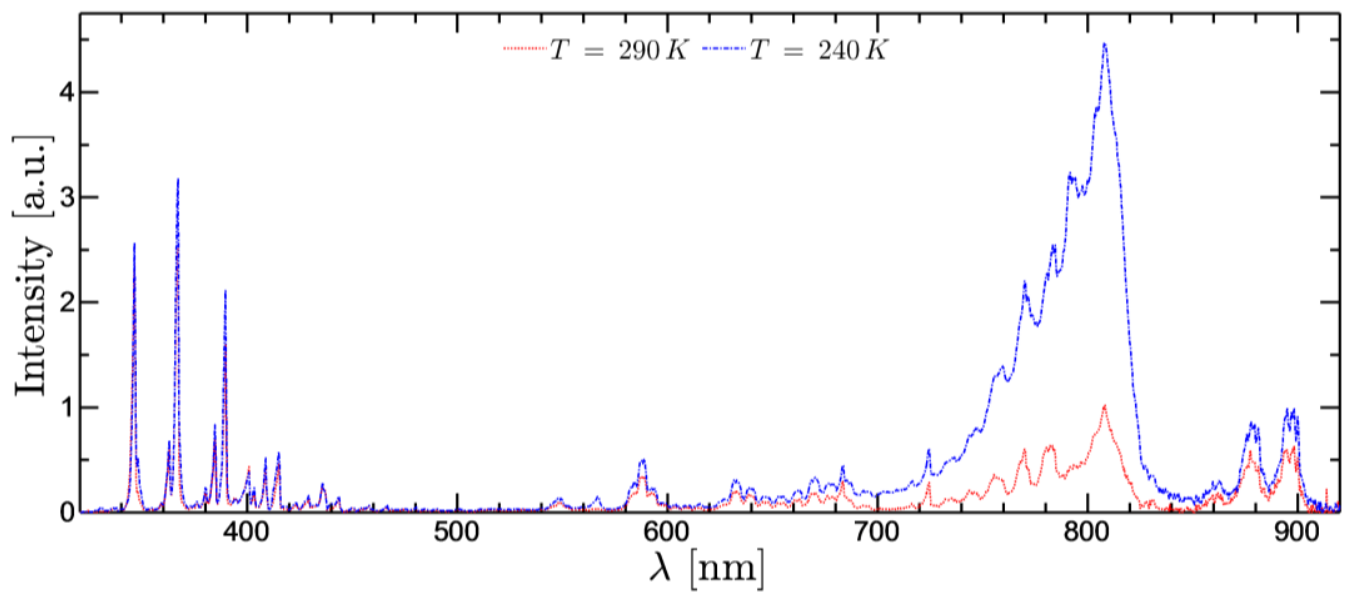

Figure 1. Calibrated, time integrated FLEET emission spectra at two temperatures.

Another aspect of the FLEET spectral theory that is relevant to the present study is the temperature dependence. Some preliminary work has shown the decay of the first negative and second positive systems to be useful as a temperature indicator at elevated temperatures. ${ }^{33}$ In the present studies, the expected temperature range was between 100 and $300 \mathrm{~K}$ (substantially reduced temperatures). Consequently, having some indication of the spectral response within this range was informative to the overall preparation of experiments. Figure 1 shows two spectralresponse-calibrated, temporally-integrated FLEET spectra taken at ambient temperature and a reduced temperature (collected and calibrated in a manner consistent with Ref. 35). The peak intensity of the emission band in the UV portion of the spectrum is found to remain largely unchanged as a consequence of the temperature difference. Though there is a theoretical temperature dependence in this portion of the FLEET spectrum associated with thermally equilibrated rotational bands, the resolution in the spectrometer was insufficient to resolve this subtle feature. In contrast, the effect of temperature on the visible and near IR (NIR) portions of the spectrum is more pronounced; an increase in the intensity throughout both portions of the spectrum is observed. As noted previously, the visible and NIR emissions result from the decay of a vibrationally-excited B state. It is theorized that this state is 
indirectly repopulated by a three-body recombination process of atomic nitrogen $\left(N\left({ }^{4} S\right)+N\left({ }^{4} S\right) \rightarrow N_{2}\left({ }^{5} \Sigma_{g}^{+}\right)+\right.$ $\left.M \rightarrow N_{2}\left(B^{3} \Pi_{g}\right)+M\right)$. Consequently, it is possible that the increased density at lower temperatures leads to a higher rate of recombination and thus the considerable increase in the observed signal in these spectral regions. A decreased signal lifetime would also be anticipated in these conditions, which was observed experimentally (see Section IV.C), though the signal was not spectrally resolved to truly assess this possibility. It should also be noted for completeness that the dramatic increase in the observed signal within $\pm 10 \mathrm{~nm}$ of $800 \mathrm{~nm}$ in Fig. 1 is likely from Rayleigh (or Mie) scattering and then would not have implications for making measurements in the current wind tunnel experiments.

\section{Experimental Program}

This section contains technical information about the test facility, experimental setups, and analytical methodologies used in the FLEET experiments and subsequent data analysis.

\section{A. Test Facility}

All FLEET experiments were carried out in the $0.3-\mathrm{m}$ Transonic Cryogenic Tunnel (0.3-m TCT), located at NASA Langley Research Center in Hampton, VA. A diagram of the major components of the facility can be found in Fig. 2. The 0.3-m TCT is a closed-loop, fan-driven wind tunnel capable of operating with both air and nitrogen $\left(N_{2}\right)$ environments at absolute total pressures ranging from 14.7 to 58 psia $\left(1 \times 10^{5}\right.$ to $\left.4 \times 10^{5} \mathrm{~Pa}\right)$. In nitrogen environments, total temperatures ranging from 104 to $320 \mathrm{~K}$ are possible - above ambient temperatures, heating is achieved through natural heat exchange with the motor assembly, while below ambient temperatures, liquid nitrogen $\left(\mathrm{LN}_{2}\right)$ is injected upstream of the primary nozzle contraction in varying degrees. In the present study, the experiments were limited to operating at total temperatures above $205 \mathrm{~K}$. Below that temperature, the FLEET signal to noise ratio diminished to an unusable level (this phenomena will be studied in future work). Additionally, the tunnel is capable of operating at Mach numbers ranging from 0.1 to 0.75 , though there is considerable uncertainty in the Mach number below 0.2 . The operating unit Reynolds numbers afforded by this facility range from $1.3 \times 10^{6}$ to $100 \times 10^{6}$ per foot $\left(4 \times 10^{6}\right.$ to $300 \times 10^{6}$ per meter), which are made possible by using various combinations of total pressures, temperatures, and Mach numbers. ${ }^{2}$

The $0.3-\mathrm{m}$ TCT features a central test section having cross-sectional dimensions of 13 in $\times 13$ in $(0.33 \mathrm{~m} \times 0.33$ $\mathrm{m}$, despite the name of the facility), which is surrounded by a sealed plenum of nominally quiescent air. Optical access to the test section is afforded by a pair of UV-grade fused silica windows. One circular window ( 8 " in diameter) resides on the outer wall of the plenum, while another ' $\mathrm{D}$ '-shaped window is mounted in the wall of the inner test section. The relative position of these two windows can be seen in Fig. 3.

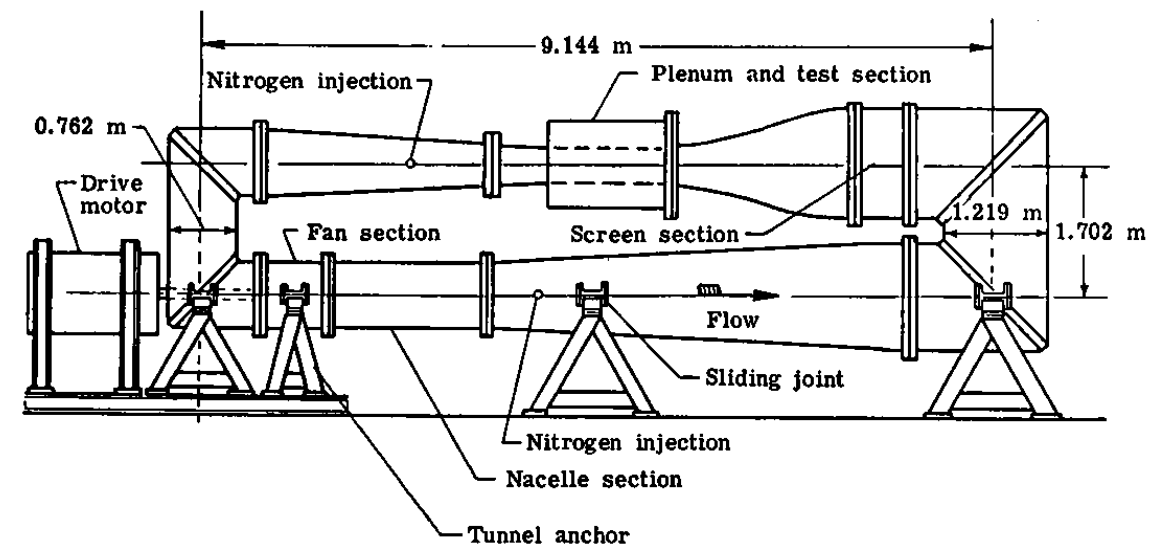

Figure 2. Schematic of 0.3-m TCT facility. From [3].

\section{B. Experimental Setup}

\section{Laser and optical systems}

The FLEET signal was generated using a regeneratively-amplified Ti:Sapphire laser system (Spectra-Physics Solstice). This laser system features a pulsed output at $1 \mathrm{kHz}$, with a center wavelength of $800 \mathrm{~nm}$, a bandwidth of $20 \mathrm{~nm}$ (FWHM), and a pulse duration of roughly $72 \mathrm{fs}$ as measured by an autocorrelator. Typical runs utilized a 
pulse energy of $2 \mathrm{~mJ}$, though this was reduced to approximately $1.5 \mathrm{~mJ}$ after passing through the optical system. The output from the femtosecond laser was first passed through an optical shutter followed by an attenuator. This arrangement allowed the laser both to be inhibited while not in use and to be adjusted for overall energy content. The beam was then diametrically expanded by a factor of 1.7 using a Galilean telescope. A series of periscopic mirror pairs was used to first, bring the beam down to the height of the test section, and second, elevate the beam between the outer pressure-shell window and the inner ' $\mathrm{D}$ '-window. The beam was focused through the test section using a 250-mm effective focal length (EFL) spherical lens, which located the FLEET signal approximately halfway through the test section. Diagrams of the optical system in immediate vicinity of the tunnel is shown in Fig. 4. The height of the beam was made adjustable using a lab jack to manually translate the upper mirror of the internal periscope and the focusing lens to the desired position. The three measurement locations are located $6 \mathrm{~mm}, 9 \mathrm{~mm}$, and $33.5 \mathrm{~mm}$ off the top wall of the tunnel and are denoted in Fig. 3 along with the coordinate system.

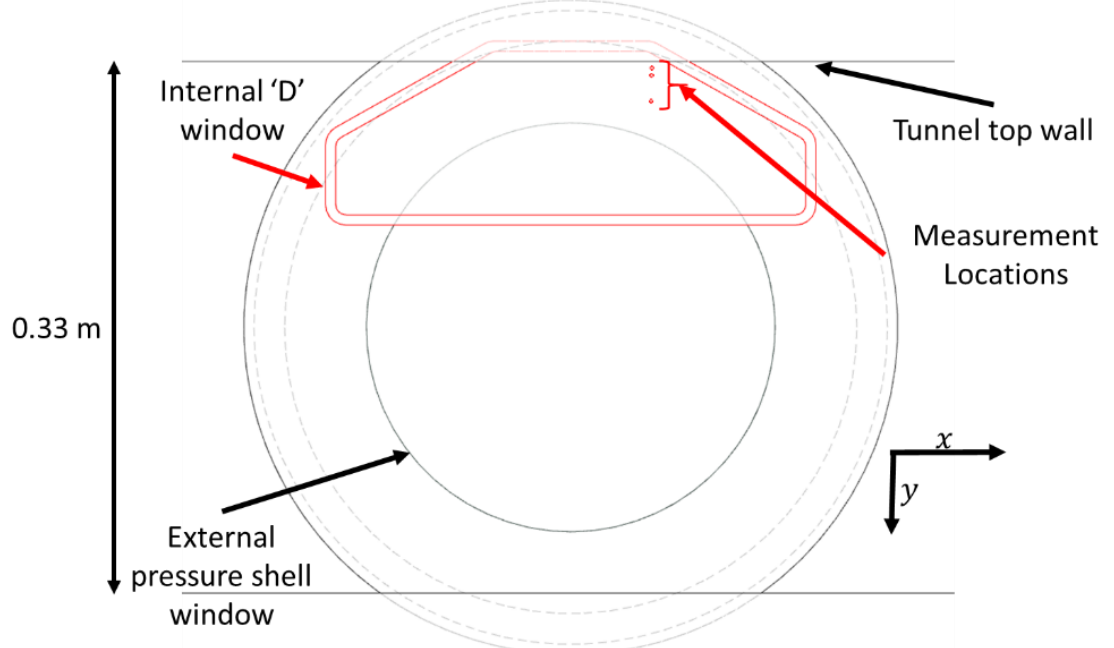

Figure 3. Schematic of test section and window layout. FLEET measurement locations and coordinate axes shown for reference. FLEET measurement locations to scale.

\section{Data acquisition systems}

Imaging of the FLEET signal was achieved using a high-speed CMOS camera (Photron FastCAM SA-Z) framing at $200 \mathrm{kHz}$. This camera lens-coupled to with an image intensifier (LaVision HS-IRO), which triggered at the same frequency as the camera acquisition with a typical gate width of $1 \mu \mathrm{s}$ and was equipped with a $135 \mathrm{~mm}, \mathrm{f} / 2$ lens, and a custom shortpass filter to block laser scatter. This equipment was determined to yield the highest quality imaging results in past work. ${ }^{36}$ The camera imaged the FLEET signal in a quasi-boresight configuration from an inclined plane elevated at approximately an $8.5^{\circ}$ angle; imaging was done through both the pressure-shell window and the internal ' $\mathrm{D}$ '-window. This arrangement was necessary due to the limited optical access in the facility. A schematic of this imaging configuration can be seen in Fig. 4. The digital resolution of the system was measured to be approximately $140 \mu \mathrm{m} / \mathrm{px}$. The camera and intensifier acquisitions were triggered using a digital pulse generator (BNC 577-8) operating in a triggered burst mode. The trigger signal was synchronized with the laser output at 1 $\mathrm{kHz}$, and typically 20-30 images were captured per burst depending on the lifetime of the FLEET signal. The first usable frame within the burst occurred approximately $320 \mathrm{~ns}$ after the laser pulse.

In addition to the camera system, an extensive facility data acquisition system was in place to measure the relevant conditions in the tunnel. This system comprised an array of static and total pressure probes throughout the facility, as well as thermocouple probes and strain gauges. These data were read into a network of facility computer systems for processing. Velocities were calculated based on static and total pressure probes and a total temperature probe in conjunction with a real-gas equation of state (Beattie-Bridgeman equation) for computing the density of the gas. An additional input to the facility data system measured when the image acquisition system was active. For each FLEET data run (typically lasting 300-400 ms or 300-400 laser pulses), a data point from the facility system was collected for use in validation and verification of the velocimetry data in post-processing. 


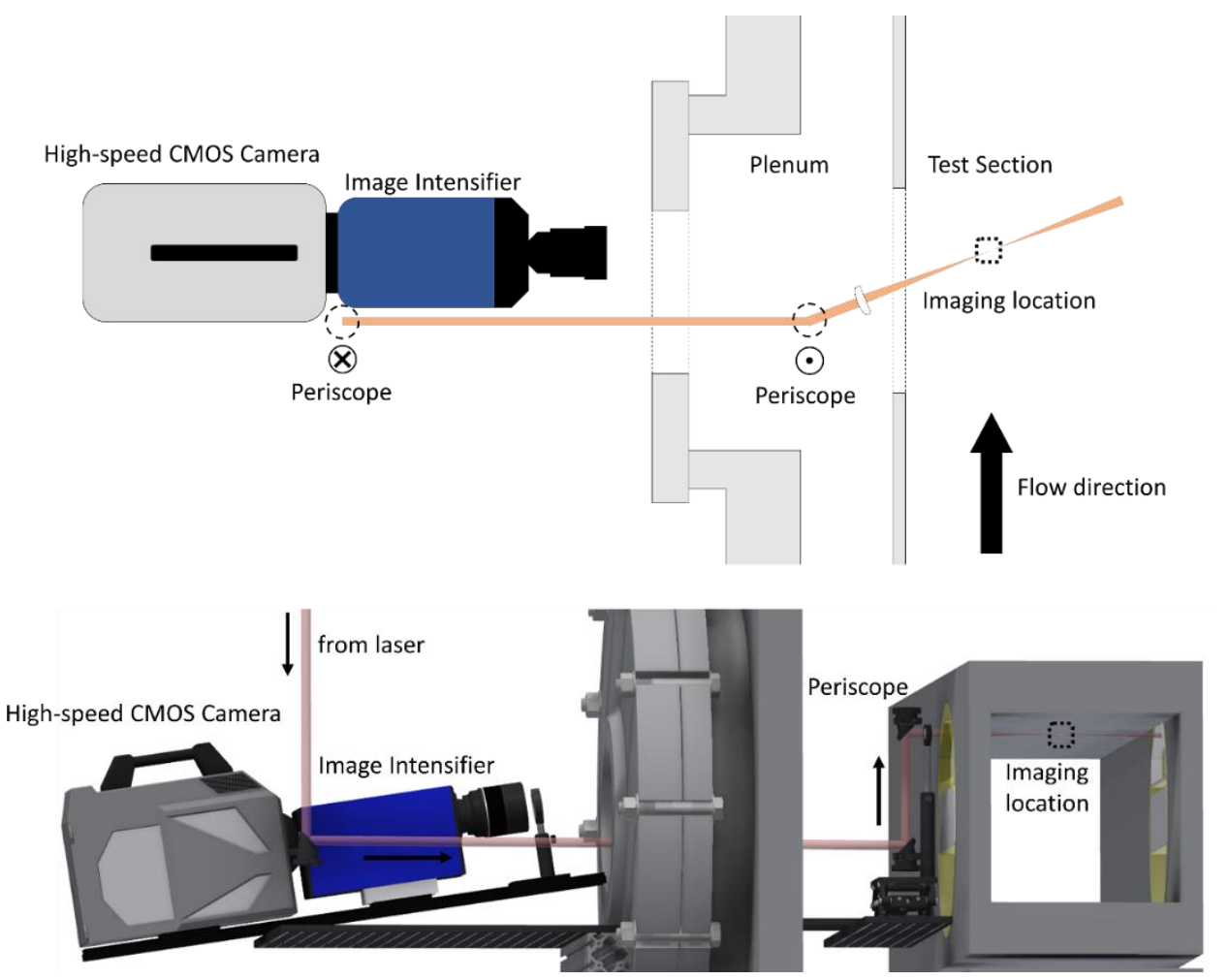

Figure 4. Diagram of FLEET imaging and optical setup. Top - overhead view; bottom - perspective view.

\section{Analytical Methods}

\section{Measurement of centroid locations}

Because of the imaging configuration, the resulting FLEET signal appeared as circular patterns in the images. These needed to be analyzed to determine the frame-to-frame displacement of the fluid. Evaluation of the positional data was done using a two-dimensional, poly-Gaussian, least-squares surface-fitting algorithm. The data were first pre-processed by making a dark-field subtraction and subsequently cropping the image to the immediate vicinity of the FLEET signal to reduce the overall processing time. While there are various methods for the accurate evaluation of velocimetry data including curve-fitting ${ }^{25}, 37$, cross-correlation ${ }^{38},{ }^{39}$, and centroid-finding algorithms ${ }^{40}$, the acquired data leant itself to the surface fitting method due to its nearly-Gaussian shape. Figure 5a shows a sample of the data acquired during these tests. The functional form of the surface-fit is given by:

$$
I(x, y)=\sum_{i=1}^{n} I_{i} \exp \left(-\left(\frac{\left(x-x_{c}\right)^{2}}{s_{x, i}}+\frac{\left(y-y_{c}\right)^{2}}{s_{y, i}}\right)\right)
$$

The parameters in Eq. (1) being fit are the values of $I_{i}$ and $s_{i}$, which vary for each surface being fit, in addition to $x_{c}$ and $y_{c}$, which are held constant across the different sub-surfaces. Typically, it was only necessary to superpose two or three surfaces in this fashion to obtain a high-fidelity fit; an example of a fit to the experimental data can be found in Fig. 5b. There were a number of advantages to this procedure over the others aforementioned. First, the output data from the algorithm were the absolute centroid locations; this is a distinct advantage over cross-correlation-based methods, in which the displacements are estimated. Particularly for burst imaging such as this, the signal-to-noise ratio (SNR) decreases with each successive image in the burst due to the finite lifetime of the FLEET signal. Consequently, if trying to estimate absolute positions for calculating Lagrangian statistics, the cumulative uncertainty in each successive frame grows rapidly as the quadratic sum of all those in the burst. While not important for two to three successive images, the current imaging method could capture up to 20 successive images, making the resultant uncertainty quite substantial. This method makes the positional uncertainties independent of previous frames. 

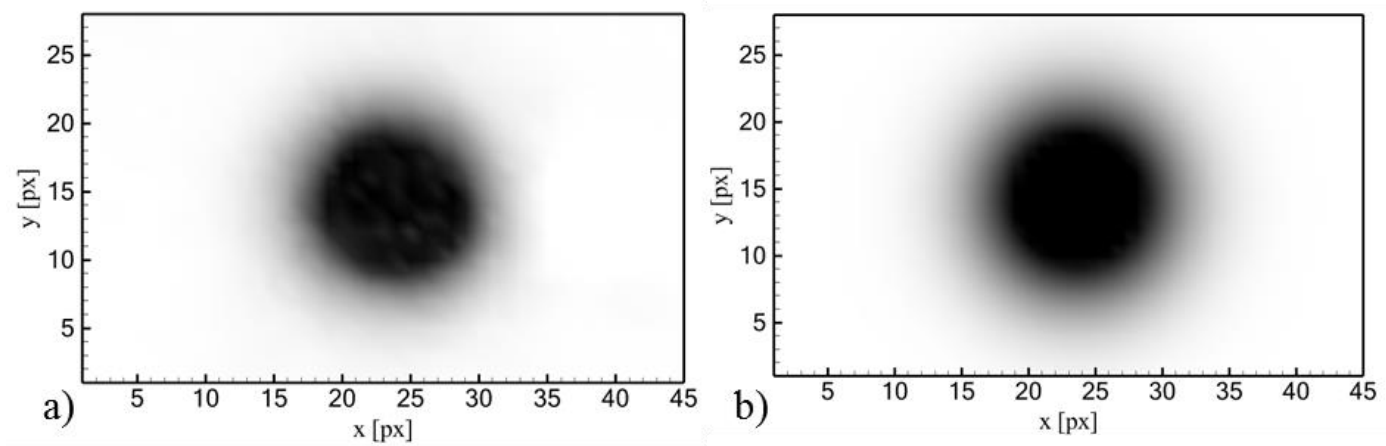

Figure 5. Comparison of FLEET signal and surface fit. a) Actual FLEET signal and b) surface fit

\section{Estimation of velocities}

Typical velocimetry data allows the velocity to be calculated by means of a finite-difference estimation of the displacement derivative. That is:

$$
v=\frac{d x_{i}}{d t} \approx \frac{\Delta x_{i}}{\Delta t}
$$

If only a single image-pair is available, little can be done to numerically improve this approximation. In the current data set, however, many subsequent centroid positions are available for use in estimating the trajectory and velocity of the FLEET signal. Owing to this, the data can be subjected to traditional particle-tracking algorithms to obtain a more accurate estimate of the desired quantities.

\section{a) Standard Method $\left(v_{1}\right)$}

Three different procedures were utilized for estimating the velocity of the FLEET signal, all of which are illustrated in Fig. 6. The first method is the traditional finite-difference method of calculating velocities, applied across subsequent time steps. For a series of $N$ data points, a maximum of $N-1$ velocities and $N-2$ accelerations can be calculated. A velocity calculated in this fashion is denoted $v_{1, i}$, where $i$ denotes the directional index (i.e., $x, y$, or $z$ ). When discussed in the context of accuracy or if a characteristic value is required for analysis, the mean of the first calculated velocities in a set is used. That is,

$$
\bar{v}_{1, i}=\frac{1}{K} \sum_{j=1}^{K} \frac{\Delta x_{i, 1 \rightarrow 2, j}}{\Delta t_{1 \rightarrow 2, j}}
$$

where $j$ in Eq. (3) is the index within a particular data set, $K$ is the total number of valid bursts within that data set, and the subscript $1 \rightarrow 2, j$ indicates the change is between the first and second images in burst $j$. This method will also be referred to as the 'standard' method.

\section{b) Precision Method $\left(v_{2}\right)$}

The next method utilized progressive time steps rather than successive time steps, wherein the velocity was calculated using Eq. (2) for the initial point and some subsequent point $n$, or $v_{2, i}=\Delta x_{1 \rightarrow n} / \Delta t_{1 \rightarrow n}$. The velocity is thus estimated at a point between points 1 and $n$, and the acceleration can be calculated by utilizing a point central to the two end points. This methodology is illustrated in Fig. 6. The implicit assumption in using this estimation is that the acceleration is effectively constant over the time interval, $\Delta t_{1 \rightarrow n}$, which is dubious if the time scales associated with any unsteadiness in the flow are of the same order of magnitude as the temporal separation. As will be assessed, this method exhibits a greater resistance to experimental noise than the first method and offers the highest measurement precision of the three methods herein utilized. The characteristic velocity associated with this method is defined in terms of the standard deviation of the velocity distribution. Specifically, the point within a burst where the standard deviation of the velocity distribution is a minimum is taken to be the characteristic velocity for the set of $K$ bursts:

$$
\bar{v}_{2, i}=\frac{1}{K} \sum_{j=1}^{K} \frac{\Delta x_{i, 1 \rightarrow n_{\sigma_{2, i}, \min , j}}}{\Delta t_{1 \rightarrow n_{\sigma_{v_{2, i}, \min }, j}}}
$$


In Eq. (4), the subscript $\sigma_{v_{2, i}, \min }$ represents the minimum standard deviation of the velocity distribution, while $n_{\sigma_{v_{2, i},} \text { min }}$ represents the index within the burst at the self-same position. In section IV, this method is referred to as the

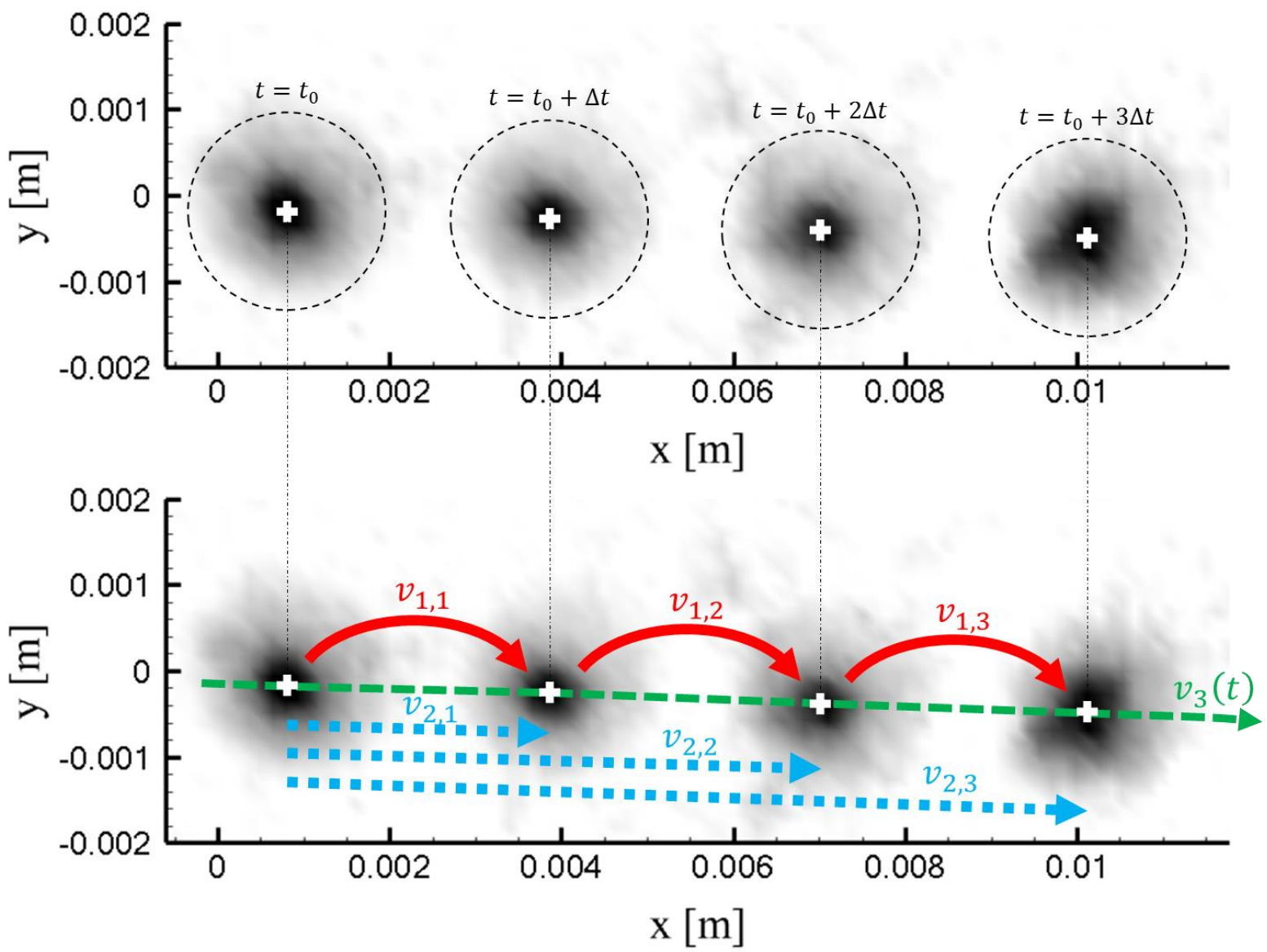

Figure 6. Diagram depicting the different methods of calculating velocity. Red - standard method, Blue precision method, and Green - polynomial method. For clarity, one of every three images are shown.

'precision' method and is denoted $v_{2, i}$. Figure 7 depicts an example time trace of this calculation method, where Fig. 7a shows the mean velocity and Fig. $7 \mathrm{~b}$ depicts the standard deviation of the velocity.

\section{c) Polynomial Method $\left(v_{3}\right)$}

The final method employs a trajectory-based estimation of the velocity and acceleration, wherein an analytic function (of time) is fit to the available position data. This formulation allows for a direct evaluation of the velocity and acceleration as a function of time. In general, a second-order (parabolic) function was fit to the experimental data (both the $x$ - and $y$-components), though a third-order polynomial was fit at the same time to ensure the estimated acceleration was nearly constant as assumed. Through this method, the position of the FLEET signal is now given by a functional form expressed as

$$
x(t)=x_{0}+v_{0} t+\frac{1}{2} a t^{2}
$$

In the context of Eq. (5), $x_{0}$ is the initial position of the FLEET signal, $v_{0}$ is the intial velocity, and $a$ is the temporal acceleration. The location of the FLEET signal was shifted such that the position in the first frame was equal to zero. Consequently, the $x_{0}$ term in Eq. (5) was generally equal to zero within the uncertainty of the fitting procedure. Figure 6 illustrates this method on sample data. Similar methods are commonplace in particle-tracking velocimetry and have shown great promise at increasing measurement precision while simultaneously reducing the potential measurement bias associated with the previous two methods, which assume no acceleration when computing the velocity from two points. ${ }^{41,42,43}$ The $v_{0}$ term of the displacement function was taken to be the characteristic velocity when evaluated on the mean trajectory, and is denoted $v_{3, i}$ or the 'polynomial' method 
throughout. Each of these three methods for calculating velocity will be evaluated in the context of the present experiment in section IV.A.
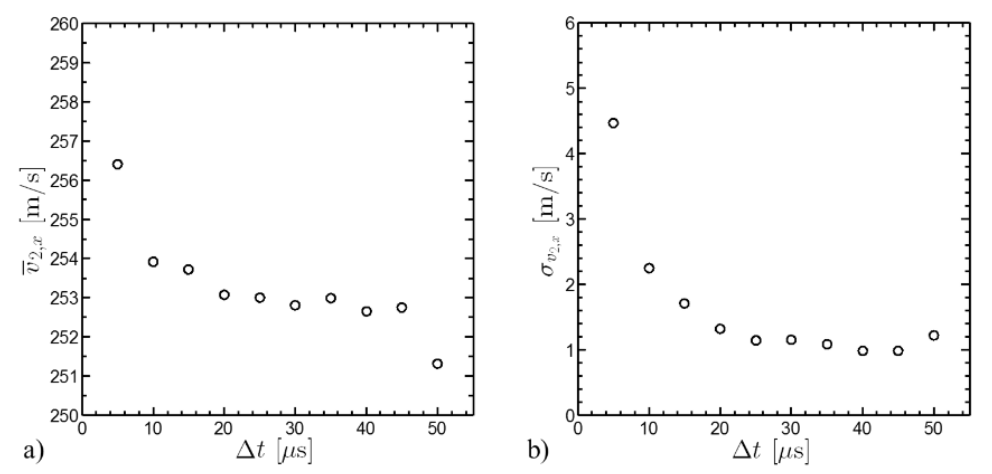

Figure 7. Example time trace of velocities calculated using progressive method. a) Mean velocity and b) standard deviation of velocity distribution.

\section{Results and Discussion}

The results for the application of FLEET velocimetry in the 0.3-m TCT are herein presented and discussed. The FLEET velocity measurements made in both quiescent air and the tunnel freestream are evaluated for their accuracy and precision. This section is followed by a cursory study of the freestream flow properties and turbulent boundary layer.

\section{A. Measurement Accuracy, Precision, and Uncertainty}

When assessing the quality of the measurements made in this facility, it became necessary to establish some general metrics of the accuracy and precision of the technique. To address the accuracy (denoted $\varepsilon_{v}$ ), a reference point was needed for comparison, which is afforded by the facility data acquisition system (DAS, for brevity). The resultant comparison for the polynomial method are shown in Fig. 8. The velocities here range from 0 for a wind-off condition up to $270 \mathrm{~m} / \mathrm{s}$ for the high end of the Mach number scale; the total temperature for all runs shown in Fig. 8 was $295 \mathrm{~K}$ (ambient), though the static temperature did vary by roughly $15 \mathrm{~K}$ over the Mach number range. Agreement is found across the entire velocity domain independent of the total pressure condition being utilized, with the mean deviation less than 0.05 percent of the DAS velocity measurements. Similar accuracies are found for the other two velocity evaluation methods as well, with the standard method having a mean deviation less that 0.5 percent, and the discrepancy in the precision method less than 1.2 percent. These results are summarized in Table 1 below. While accuracies for the various MTV techniques are not commonly presented in the literature (most present the measurement precision only), those which are reported vary broadly. Sijtsema et al., in utilizing APART velocimetry, found accuracies ranging from 5 percent to 13 percent depending on the flow being studied. ${ }^{29}$ The acetone MTV by Lempert et al. yielded accuracies of approximately 3.5 percent in application to supersonic microjets. ${ }^{44}$ In general the existing evaluation methods yield accuracies lying in the sub-pixel displacement range of 0.05 to $0.1 \mathrm{px}^{37}, 38,39$, which yield varying degrees of accuracy depending on the flow and image magnification in addition to the temporal separation used in the data acquisition. The greatly improved measurement accuracy in these studies arises from several factors. First, as described in Section III.C.1, the FLEET data lent itself to the surface fitting algorithm that was utilized. Additionally, the signal-to-noise ratios were typically quite high in these data, increasing both the accuracy and precision of the measurements. Since the operating pressures within the test section were high (1 to $4 \mathrm{~atm}$ ) compared to some of the other works mentioned above, the diffusivity of the nitrogen was lower, allowing the FLEET signal to remain cohesive (and thus permit an accurate surface fitting) for a longer duration. Furthermore, the data that were acquired consisted of not a single image pair, but a sequence of 10 to 15 images. By utilizing all of these data, not only are the measured accuracies increased as demonstrated, but the dynamic range of the measurement is also extended. Thus, the accuracy is preserved over the range of velocities herein presented and - in all likelihood - to lower velocities as well. In conjunction with statistically significant ensembles of data and well-controlled/characterized flow conditions within the 0.3-m TCT facility, these characteristics contributed to the relatively high accuracy of the velocity data. 


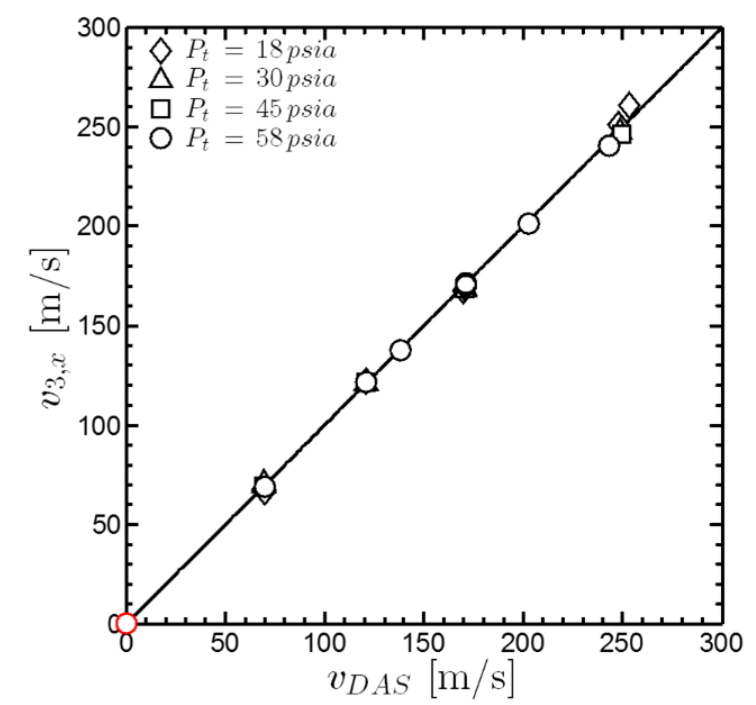

Figure 8. Comparison of velocities measured by the facility DAS and those measured by FLEET. Only the polynomial method results are shown for clarity.

For completeness, several points needs to be discussed in regard to the accuracy of the velocity measurements. As mentioned, the velocity comparison were all made with respect to the DAS, whose probes used to measure the freestream velocity were located upstream of the FLEET measurement location by roughly $0.75 \mathrm{~m}$. Thus, there is an expectation that the flow accelerated by some amount by the measurement location. However, the growth of the boundary layers was counteracted by the position of the flexible walls on the floor and ceiling of the test section. ${ }^{2}$ Consequently, the degree to which the calculated velocities are affected is not entirely clear. However, based on DAS wall pressure measurements along the length of the test section, there is a 0.5 to 2 percent uncertainty in the DAS velocities. Thus, the precise extent to which the measured accuracies are affected is unclear, but it does not extend beyond this uncertainty mark.

A final observation to discuss regarding the accuracy of the FLEET velocity measurements was a measured deceleration in the stream-wise velocity present in most runs. A mean deceleration was observed in the FLEET signal when using both the standard and polynomial evaluation methods; the effect accounted for perhaps a 1 to 2 $\mathrm{m} / \mathrm{s}$ change over the distance of the measured trajectories. This deceleration is the reason for the decreased accuracy in the precision method listed in Table 1, as it tended to bias those velocities toward a lower value, while the other two measurements accounted for the deceleration in some capacity. The cause(s) of this phenomenon is not clear at present. It is possible that the perturbation of the flow caused by the FLEET excitation (see Ref. 45) has caused an (initial) positive bias to the velocity, and the observed acceleration is a response to said perturbation. Alternatively, the de-warping algorithm utilized in the spatial calibration of the FLEET data might have been insufficient to remove any perspective distortion in the images, though such distortion was only minimally present. While worth noting this observation, the effect of the acceleration on the overall quality of the measurements was quite minimal, and the influence on the accuracies were less than one percent across the entire velocity domain.

The precisions of the measurement methods are summarized in Table 1. The precisions (marked as $\sigma_{v}$ ) for each velocity evaluation method are defined as the standard deviation of the calculated velocity distribution in static gas. For the standard method, the velocity distribution considered was the one generated between the first two frames in a burst. For the precision method, the standard deviation for all available $1 \rightarrow n$ evaluations was calculated and corrected for disparate sample sizes, and then the minimum was taken to be the true precision. Typically, this occurred in the $7^{\text {th }}$ or $8^{\text {th }}$ frame before increasing again, though it did vary from run to run. Finally, with the polynomial method the probability distribution of $v_{o}$ was used in the evaluation of the precision. In general, it can be seen that the value of the precisions are quite small, ranging from $0.53 \mathrm{~m} / \mathrm{s}$ for the precision method to a few meters per second.

A few notes regarding the estimations: these precision values were calculated based on wind-off data in air; the gas is nominally static. On the time scales of each burst $(<125 \mu \mathrm{s})$, buoyancy and thermal relaxation effects can be considered negligible as they occur on much longer $(O(1 \mathrm{~ms}))$ and much shorter $(O(10 \mathrm{~ns})){ }^{45}$ time scales, respectively. Thus, any tabulated velocities used in calculating the precision are a result of scatter in the experimental data rather than any true velocity present in the flow. However, since the laser is being pulsed at $1 \mathrm{kHz}$ 
and the gas is not moving between pulses, there is the possibility of contamination from dissociative products from previous pulses reducing the lifetime and intensity of the FLEET signal, which might adversely affect the precision.

${ }^{36}$ Addressing the uncertainties in the mean velocities $\left(\delta_{\bar{v}}\right)$ in Table 1 , it was observed that all velocity evaluation methods yielded uncertainties less than $1 \mathrm{~m} / \mathrm{s}$. The uncertainties are calculated by the formula $\delta_{\bar{v}}=\sigma_{v} \sqrt{p /(n K)}$, where $K$ is the number of samples constituting the distribution (181 in this static set), $n$ is the number of sample points considered in the fit $(n=2$ for the standard and precision methods, and $n=11$ for the polynomial method, though it varies from run to run), and $p$ is the number of degrees of freedom in the fit ( $p=1$ for precision and standard methods, and $p=2$ for the polynomial method). The polynomial fitting method and precision methods yielded similar uncertainties, while the standard method had a larger uncertainty due to the broader distribution of calculated velocities. These measurements indicate that the FLEET velocimetry technique is capable of making high-fidelity measurements of velocity over a wide range of operating pressures in the $0.3-\mathrm{m}$ TCT facility. The effects of running at significantly reduced temperatures will be discussed in section IV.B.

As a final examination of these static velocity measurements, the typical frequency response of each evaluation methods is summarized in Table 1. The different evaluation methods each require a varying number of points to attain their maximum precision and greatest degree of accuracy. The standard method utilizes only two successive points, which with the present measurement system required a temporal separation of only $5 \mu$ s, independent of the run. Thus, the typical frequency response in these measurements was the full $200 \mathrm{kHz}$ acquisition rate of the imaging system. The precision method, which utilized two points with varying degrees of separation, typically attained its tightest precision after 7 to 8 data points as mentioned previously. This method consequently limited the resolvable fluctuations in velocity to approximately $200 / 7=29 \mathrm{kHz}$. Finally, the polynomial method utilized a similarly differing number of points as the precision method. However, variations in the velocity are still observable within the stencil used in the evaluation provided they can be described by a constant acceleration. In this way, the temporal response is limited to the time separation between the minimum number of points used in the fitting procedure, which is 4 (nd 4 points yields three $\Delta t$ 's) when considering the verifying cubic fit performed on the data. Thus the frequency response is limited to approximately $200 / 3=67 \mathrm{kHz}$.

Table 1: Accuracy, precision, uncertainty in the mean, and typical frequency response of FLEET velocity measurements measured in quiescent air

\begin{tabular}{lcccc}
\hline \hline Method & $\varepsilon_{\bar{v}}(\%)$ & $\sigma_{\bar{v}}(\mathrm{~m} / \mathrm{s})$ & $\delta_{\bar{v}}(\mathrm{~m} / \mathrm{s})$ & $f(\mathrm{kHz})$ \\
\hline Standard $\left(v_{1}\right)$ & 0.39 & 2.34 & 0.13 & 200 \\
Precision $\left(v_{2}\right)$ & 1.13 & 0.53 & 0.03 & 29 \\
Polynomial $\left(v_{3}\right)$ & 0.03 & 0.96 & 0.03 & 67 \\
\hline
\end{tabular}

\section{B. Freestream and Boundary Layer Measurements}

A range of different freestream conditions were probed to determine both if the FLEET technique was sufficiently sensitive to detect physical trends in the flow and to assess the effects that the varying conditions would have on the signal quality and the ability assess the velocity. Additionally, the FLEET probe volume was coarsely traversed through the tunnel top-wall boundary layer to assess the effects of the probe volume size and technique sensitivity on determining the Lagrangian and Eulerian statistics of the flow in these regions. 


\section{Freestream conditions}

The measured freestream velocity is presented as function of the tunnel static temperature in Fig. 9; these values represent the full range of total pressures presented in Fig. 8. It can be seen that the measured velocities adhere very closely to the velocities predicted by isentropic flow theory for the nominal Mach numbers. The implication of this behavior is that the FLEET technique is not only sufficiently sensitive to resolve this physical trend in the data, but the high degree of accuracy observed at ambient temperatures was maintained as the temperature was decreased by nearly $100 \mathrm{~K}$. It should be noted here that the majority of the observable spread in the velocity measurements does not arise from experimental scatter, but rather the variability in the set-point conditions within the wind tunnel facility. That is, the true Mach number may vary from the nominal condition by as much as 0.02 due to limitations in the tunnel drive system, which is why these points lie off the predicted curves. In spite of this minor imprecision in the tunnel conditions, the measured velocities are able to capture the general physics of the flow accurately, over a wide range of tunnel operating conditions encompassing the full range of Mach and total pressure conditions and static temperatures down to $205 \mathrm{~K}$.

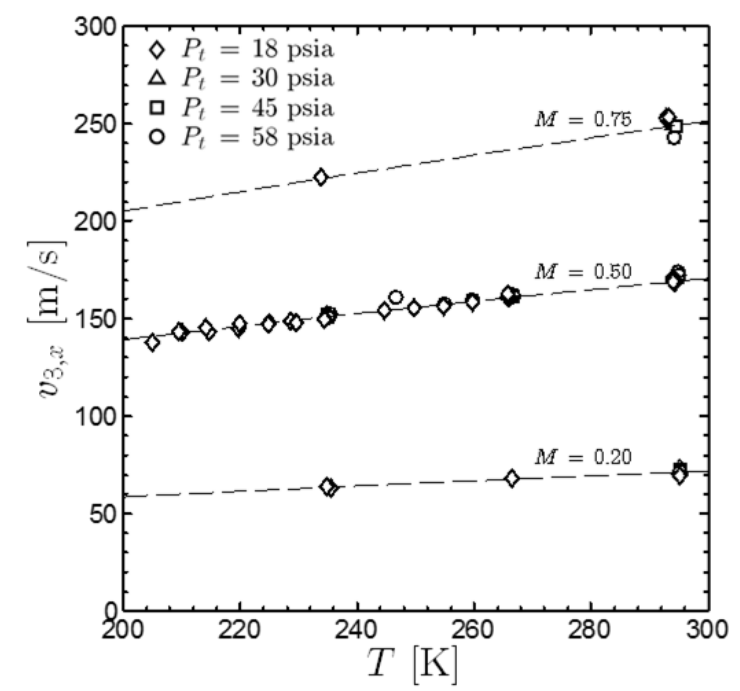

Figure 9. Measured FLEET velocity as a function of static temperature. Dashed lines represent set-point Mach numbers as labeled.

The freestream velocity fluctuations are presented in Fig. 10 as a function of the Mach number. In this context, the fluctuations are defined as the standard deviations of the measured velocity probability distributions. There is a definitive trend of decreasing fluctuation magnitude as the Mach number is increased. This is a consequence primarily of the FLEET signal lifetime, which appeared to increase with Mach number in these studies (another phenomenon that will be investigated in future work). Despite this trend, it must be noted that the fluctuations in general are less than 1 percent of the freestream velocity, which is consistent with previous measurements of the velocity fluctuations in this facility based on $\mathrm{LDV}^{5}$ and considerably lower than measurements made with dynamic pressure probes, where the inferred velocity fluctuations were between 1.8 and 3 percent. ${ }^{46},{ }^{47}$ Another apparent trend (or lack of trend) is that the magnitude of the fluctuations is largely independent of the freestream Reynolds number, represented by the different total pressure conditions. The reason for this observation is that the magnitude of the velocity fluctuations lie primarily below the observed precision in the measurement, denoted by the dashed line in Fig. 10 (for plotting, the constant-valued precision values for $v_{3}$ calculated in air have been divided by the theoretical freestream velocity at the denoted Mach numbers). The primary scatter of the measured fluctuations can be seen to lie below this line, with the exception being the lowest Mach number that was subject to the lifetime limitation as previously mentioned. Consequently, it is not possible to discern trends in this context. However, it can be said that the fluctuations are generally small enough to lie within the precision of the measurement, which are less than 1 percent across the entire observed range of Mach numbers. This observation does require some qualification; the precision measurements were made in quiescent air. In nitrogen, the signal-to-noise ratios were substantially higher, which corresponded directly to an increased precision (i.e. smaller magnitude). Moreover, the gas was not static, and thus not subject to dissociative contamination from previous laser pulses. Thus, it is not surprising to find the velocity fluctuations here to lie within the precision value calculated in air, particularly given their small magnitude. 


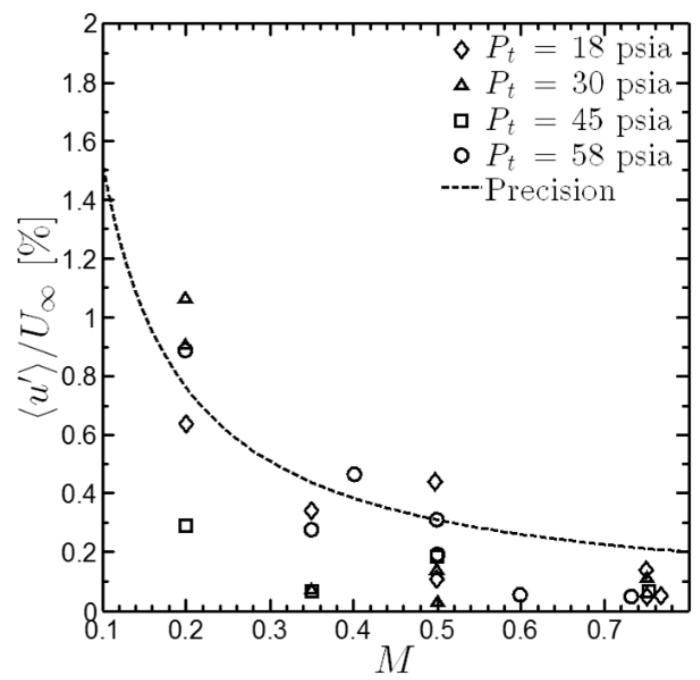

Figure 10. Magnitude of freestream velocity fluctuations as a function of Mach number. Dashed line represents the measured precision.

\section{Boundary layer}

Two measurement points were sampled within the tunnel top-wall boundary layer, located $9 \mathrm{~mm}$ and $6 \mathrm{~mm}$ from the top wall. While both of these sampling locations lie within the outer wake region of the boundary layer, the velocity was reduced and turbulence intensity increased sufficiently (compared to the freestream) to assess FLEET velocimetry. Several pathlines (alt. particle paths or trajectories) sampled with the FLEET signal at these two measurement locations as well as the freestream are depicted in Fig. 11. Focusing on the first measurement location at $y=9 \mathrm{~mm}$ shown in Fig. $11 \mathrm{~b}$, it can be seen that there is notable variability in the particle trajectories, specifically in terms of the total path length. This trend is most visible at the downstream end of the visible domain (obtained from the $11^{\text {th }}$ image), where the accrued deviations cause the greatest spread in the data and also by comparison with the trajectories measured in the freestream (Fig. 11a). It can be seen that the trajectories are relatively straight; that is, if the FLEET spot is initially moving parallel to or slightly away from the wall, it continues to do so. The particle paths measured in the freestream also possess marked parallelism, which is absent from those measured at the $9 \mathrm{~mm}$ location. Despite this unsteady behavior, the mean trajectory (given by the bold red line) trends (slightly) in the positive wall-normal direction, which is consistent with the slow growth of the boundary layer and displacement thickness. Another trend obviated by Fig. 11b is the variability in the initial FLEET spot position, whose magnitude is roughly $\pm 0.3 \mathrm{~mm}$. This artifact is a consequence of the low-frequency vibrations within the $0.3-\mathrm{m}$ TCT facility, which caused the focusing optics to vibrate. At present, it will be sufficient to say that this effect has caused an uncertainty in the position of the sampling location but has not influenced the uncertainty in the measured velocities and accelerations.

The second measurement location at $y=6 \mathrm{~mm}$ shows more notable variability in particle paths compared to the previous location; similar trajectories are depicted in Fig. 11c. There is substantial variation between the individual FLEET trajectories; they appear far more unstable than at the previous measurement location and certainly than in the freestream. This trend manifests as the somewhat erratic behavior of the FLEET spot, with (for example) some traces sweeping upward before turning back down. Such measurements are not well-fit by a polynomial containing a constant acceleration (or a single velocity for that matter), particularly in the wall-normal direction. Consequently, the polynomial method is not an appropriate method for this region of the flow if used to describe the entire trajectory. Furthermore, the motion of the FLEET spot is rather continuous, suggesting that the behavior is representative of the underlying flow rather than experimental scatter. This observation lies in stark contrast to the 9 $\mathrm{mm}$ measurement location and the freestream, where the behavior of the paths was relatively predictable given the initial trajectory (see Fig. 11a and 11b). The particle paths seen in Fig. 11c also represent a broader range of velocities compared to the other two measurement positions. This trend is most obvious when comparing the location of the FLEET spots at the different time steps (successive stream-wise positions). In contrast to Fig. 11b, where the scatter between spots grows rather slowly with time, the spread makes it difficult to distinguish individual 
time steps after 3 or 4 frames. This behavior is suggestive of a more turbulent flow with larger fluctuations
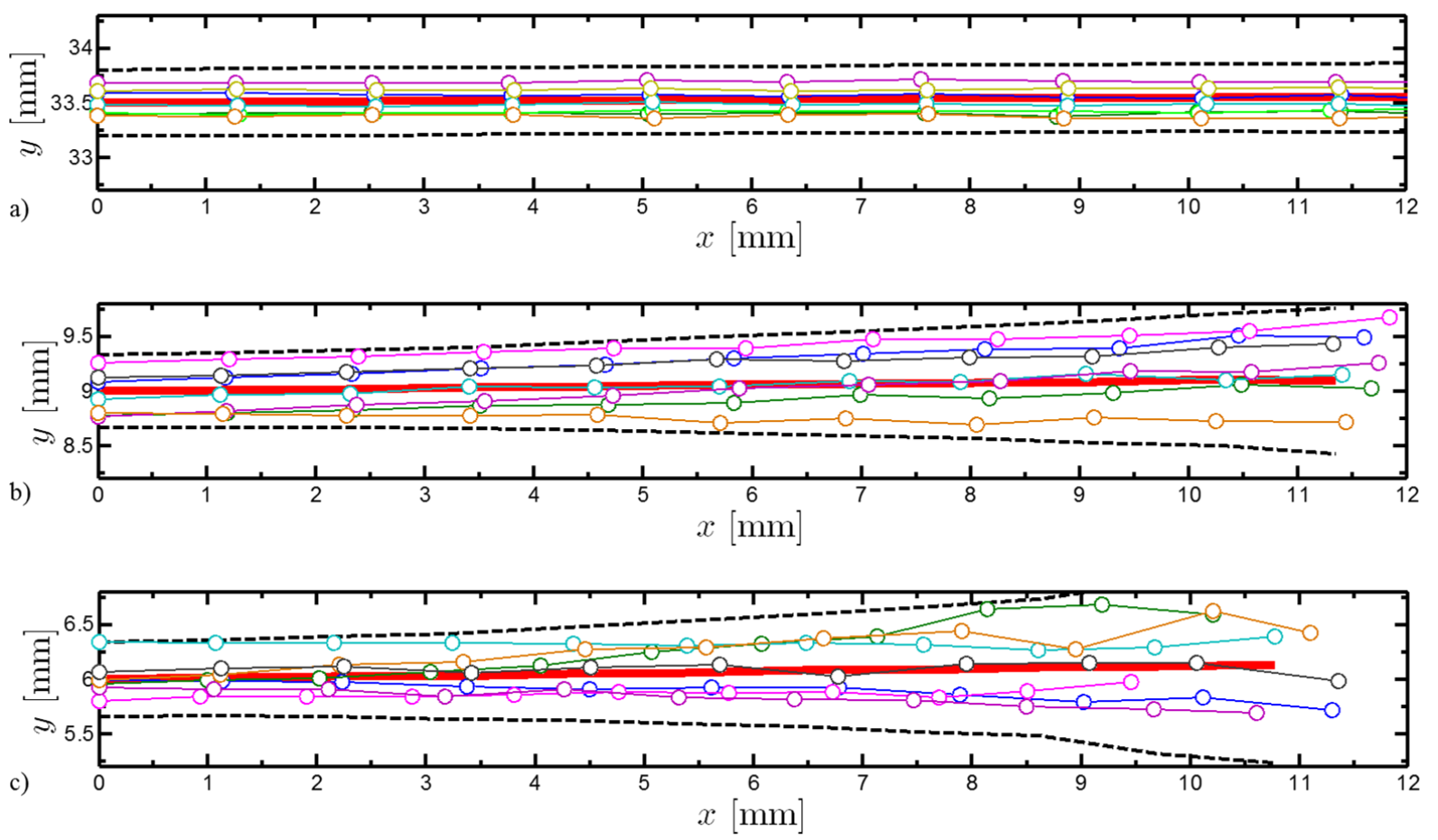

Figure 11. Sample particle paths at two boundary layer measurement locations and the freestream. a)

Measurement location in freestream $(y=33.5 \mathrm{~mm}), \mathrm{b})$ measurement location centered at $y=9 \mathrm{~mm}$, and c) measurement location centered at $y=6 \mathrm{~mm}$. Solid red line indicates the mean trajectory at each measurements location, while dashed black lines indicate the $2 \sigma$ bounds of the trajectory.

compared to the outer measurement location.

The mean velocities at the three measurement locations are depicted in Fig. 12 for the 58 psia total pressure condition (the trends also hold for the lower pressure cases); the velocities have been calculated with the precision method over a fixed number of frames/images to ensure a fair comparison of the statistics and to prevent any biasing due to a poor fit quality using the polynomial method. In Fig. 12, the effects of the varying time step can be seen to have a minimal influence on the mean velocities at any of the measurement locations with the maximum deviation at a given measurement point less than 2 percent of the mean. For comparison, a representative log-law fit for the boundary layer profile utilizing a van Driest transform (see Ref. 48) has been plotted for each of the respective Mach numbers. Though the velocity was only measured at three locations, the corresponding fit for each of the Mach numbers agreed within the positional uncertainty at each point. Maximum deviations from these theoretical profiles were roughly 4 percent. Nonetheless, the FLEET velocimetry seems to have captured the general boundary layer physics. A less coarsely sampled run would be required to make any definitive statements about the overall accuracy of the technique through the boundary layer.

As a final assessment of the FLEET measurements, the RMS velocities at the same wall-normal locations are shown in Fig. 13 for the 58 psia total pressure condition. The values have again been calculated based on the precision method, and are presented in Fig. 13 for different time steps. The variability observed in the particle paths shown in Fig. 11 are here manifest as a greater RMS velocity; this trend is seen to hold for all Mach numbers. Unlike the mean measurements presented in Fig. 12, the size of the time step was found to have a strong influence over the magnitude of the fluctuations at the lowest Mach number. At the $9 \mathrm{~mm}$ position, the Mach 0.5 and 0.75 cases show the magnitude of the fluctuations to be roughly 3.75 percent of the freestream velocity, independent of the number of time steps involved. Likewise, at the $6 \mathrm{~mm}$ position, both of the higher Mach numbers presented have a value of approximately 4.25 percent. The Mach 0.2 cases show a decremental change in the RMS velocities as the size of the time step is increased, though they appear to asymptote to 4.75 and 5.0 percent at the $9 \mathrm{~mm}$ and $6 \mathrm{~mm}$ positions, respectively. This behavior is suggestive of experimental noise associated with a lower signal-to-noise ratios at the lower Mach number condition rather than a smoothing out of the fluctuations by the evaluation method. 
Based on velocity scalings from the DNS data of Duan et al. ${ }^{49}$ we estimate the theoretical velocity fluctuations at the $9 \mathrm{~mm}$ position to be between 4.8 and 5.5 percent of the freestream velocity, while at the $6 \mathrm{~mm}$ position they would
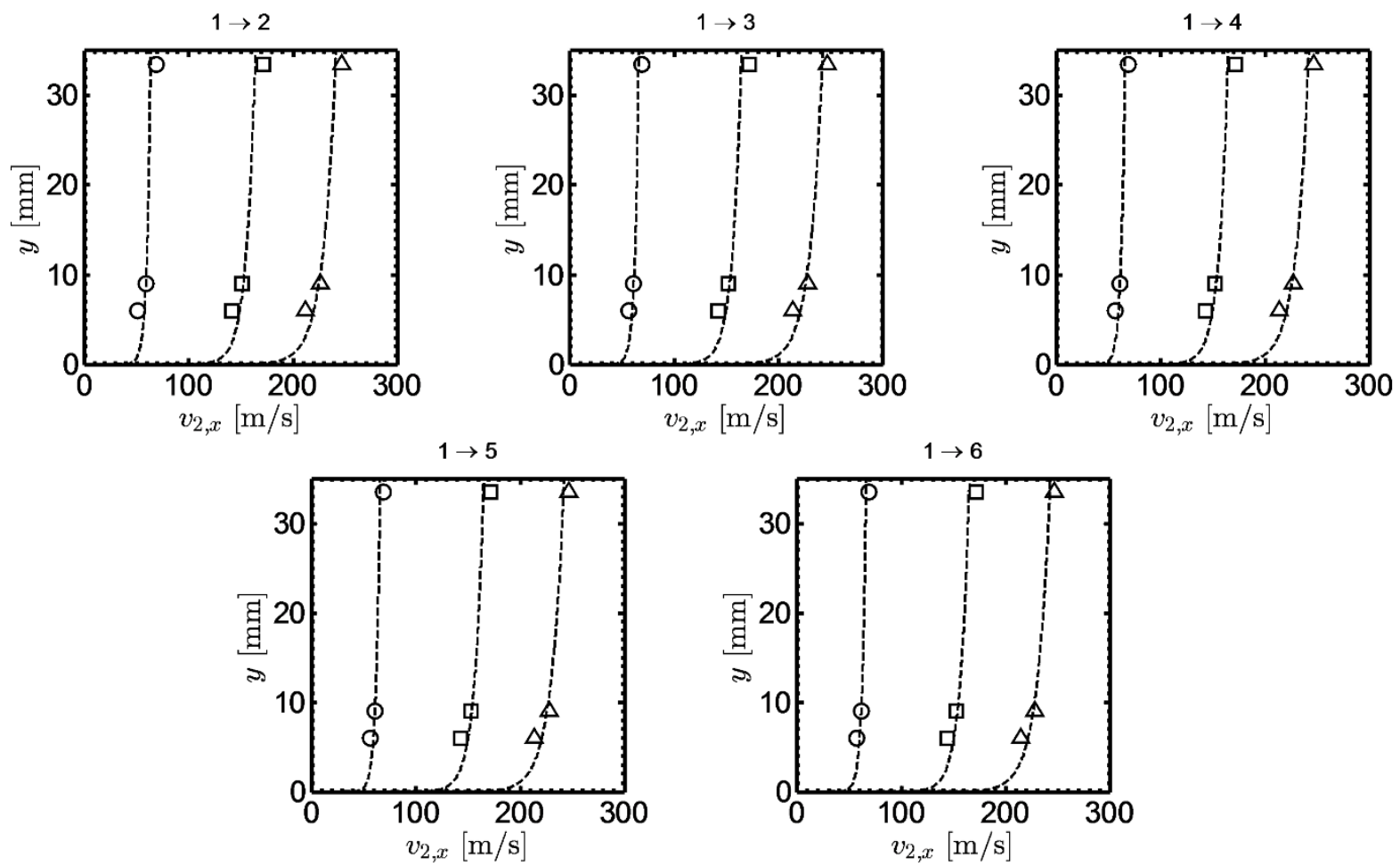

○ $M=0.20$ 口 $M=0.50 \Delta M=0.75$

Figure 12. Mean velocity profiles measured in boundary layer. Total pressure is $58 \mathrm{psia}$. Titular labels represent the frames used in their calculation

likely reside between 7 and 8 percent. By comparison, the velocity fluctuations measured by FLEET are lower than the simulations predict, but are still on the same order of magnitude. It is possible that this discrepancy is a manifestation of the disturbance to the flow that FLEET has been shown to cause. That is, the thermal perturbation it imposes (several hundred Kelvin temperature increase through the volume of the FLEET signal ${ }^{33}$, ${ }^{45}$ ) and the resulting density change can act as a damper to fluctuating motions. This possibility could explain why the measured values of the velocity fluctuations are lower than those predicted by simulations. Another possible cause of this discrepancy is

the size of the measurement volume; the FLEET signal was approximately $1 \mathrm{~mm}$ in diameter and an estimated $2 \mathrm{~mm}$ in length. It is likely that some of the fluctuations present in this portion of the boundary layer occurred within the confines of the measurement volume and would not have been resolved by the present imaging system. Note that this mm-scale spatial resolution of the FLEET signal is an artifact of how the measurement had to be implemented because of the limited optical access in the facility (exciting and detecting through a single window) and not a limitation of the FLEET technique itself, which has been used to measure with $0.1 \mathrm{~mm}$ spatial resolution. ${ }^{24}$ It is unclear whether these effects can be counteracted by any numerical efforts or utilizing data with a higher signal-tonoise ratio, though the present efforts suggest that it might be a limitation of the experimental configuration used. More data focused specifically on measuring fluctuating velocities would be required to make any definitive causal statements. Despite this observed discrepancy, the expected dependence on the Mach number is preserved in the FLEET data; the strength of the fluctuations relative to the freestream velocity increases as the Mach number is decreased. 


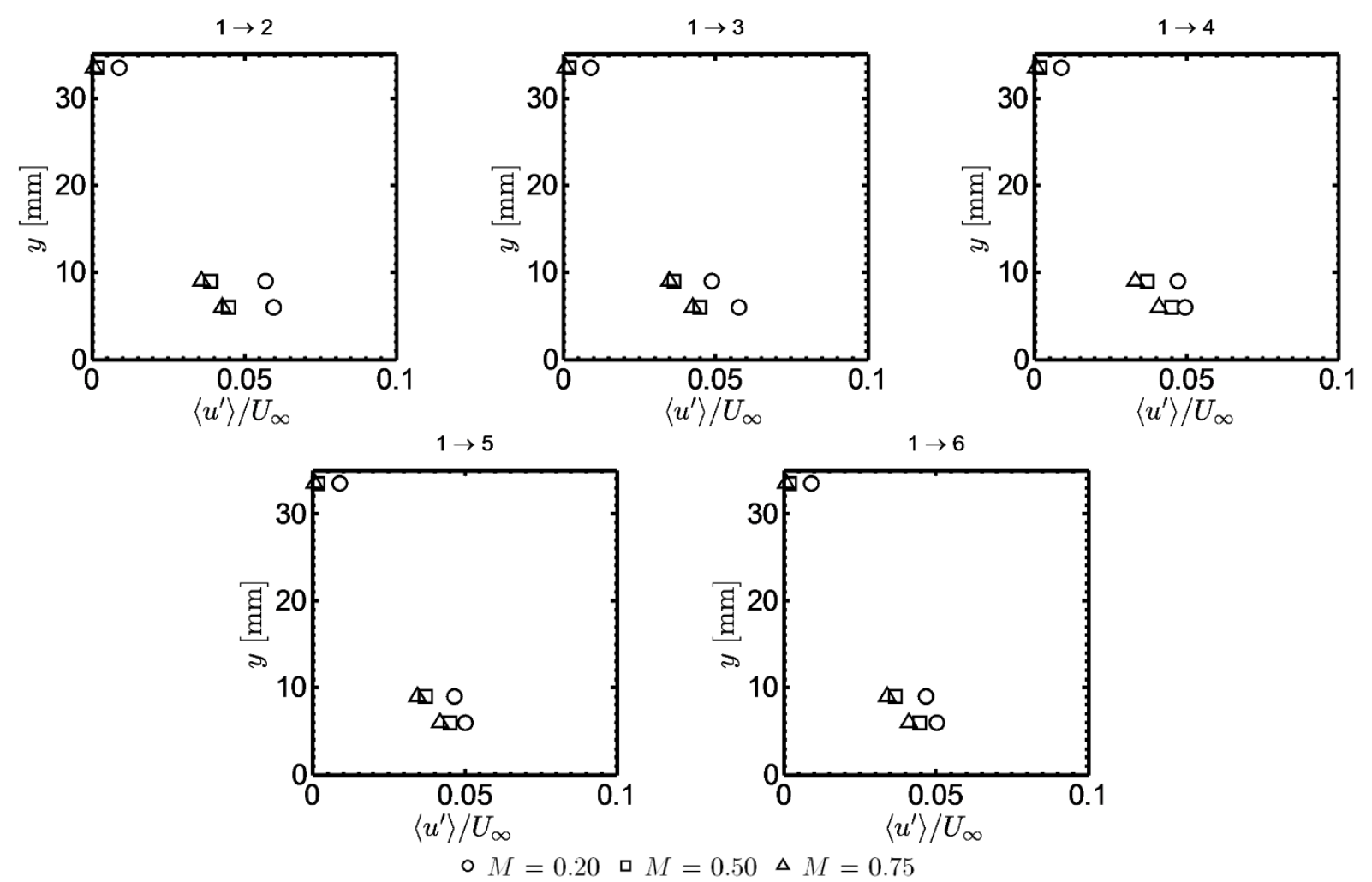

Figure 13. RMS velocity profiles measured in boundary layer. Total pressure is 58 psia. Titular labels represent the frames used in their calculation

\section{Conclusions}

FLEET velocimetry has been successfully demonstrated in NASA Langley's $0.3-\mathrm{m}$ Transonic Cryogenic Tunnel - the first application of FLEET in a large-scale test facility. The accuracy and precision of the FLEET velocity measurements were determined in the tunnel freestream and in quiescent air using three different data analysis methods for determining the velocity. These methods each exhibited a high degree of accuracy, with the lowest accuracy still within approximately 1 percent of the velocities measured by the tunnel data acquisition system. Measurement precisions in the data were better than $1 \mathrm{~m} / \mathrm{s}$ for two of the three techniques examined. The typical frequency response of each evaluation method varied, but generally showed lower frequency response as the measurement precision was increased. The velocity measurements within the tunnel freestream were able to capture the physical dependence of the velocity on the tunnel static temperature, showing utility over a $100 \mathrm{~K}$ temperature range. Within the boundary layer, the observed virtual pathlines were indicative of the increasingly unsteady behavior with decreasing wall-normal distance, while mean velocity measurements showed strong agreement with theoretical velocity profiles. The RMS velocity profiles, which were evaluated using varying degrees of temporal separation, obeyed the physical dependence on the Mach number of the flow, but were shown to differ from simulations by a few percent of the freestream velocity.

\section{Acknowledgments}

The authors would like to thank the facility operations team at the $0.3-\mathrm{m}$ Transonic Cryogenic Tunnel facility including Wes Goodman, Mike Chambers, Karl Maddox, and Cliff Obara among others. Their contributions allowed these experiments to be conducted efficiently, and they were a pleasure to work alongside for the duration of these tests. Additionally, Stephen Jones was an invaluable part of the team in the construction and implementation of the testing apparatus. Finally, the contributions and moral support of Michael Button from the George Washington University, Brett Bathel and Jennifer Inman from NASA Langley Research Center, and Sukesh Roy 
from Spectral Energies, LLC were greatly appreciated. This work was funded by the NASA IRAD Program with Marty Waszak at Program Manager and also the NASA SBIR Program, contract number NNX14CL74P.

\section{References}

1 Wahls, R. A. "The National Transonic Facility: A Research Retrospective," 39th AIAA Aerospace Sciences Meeting and Exhibit, Reno,NV, 2001, pp. 1-28.

2 Ladson, C. L. and Ray, E. J. Evolution, Calibration, and Operational Characteristics of the Two-Dimensional Test Section of the Langley 0.3-Meter Transonic Cryogenic Tunnel; NASA Technical Paper 2749: Hampton, VA, 1987.

3 Snow, W. L., Burner, A. W., and Goad, W. K., "Image Degradation in Langley 0.3-Meter Transonic Cryogenic Tunnel," NASA Technical Memorandum 84550, 1982, pp. 1-25.

4 Snow, W. L., Burner, A. W., and Goad, W. K., "Improvement in the Quality of Flow Visualization in the Langley 0.3-Meter Transonic Cryogenic Tunnel," NASA Technical Memorandum 87730, 1987, pp. 1-24.

5 Gartrell, L. R., Gooderum, P. B., Hunter, W. W., and Meyers, J. F., "Laser Velocimetry Technique Applied to the Langley 0.3-Meter Transonic Cryogenic Tunnel," NASA Technical Memorandum 81913, 1981, pp. 1-35.

6 Honaker, W. C. and Lawing, P. L., "Measurements in the Flow Field of a Cylinder with a Laser Transit Anemometer and a Drag Rake in the Langley 0.3-m Transonic Cryogenic Tunnel," NASA Technical Memorandum 86399, 1985, pp. 1-24.

7 Hunter, W. W. and Foughner, J. T. "Flow Visualization and Laser Velocimetry for Wind Tunnels," NASA Conference Publication 2243, Hampton, VA, 1982.

8 Fey, U., Engler, R. H., Egami, Y., Iijima, Y., Asai, K., Jansen, U., and Quest, J. "Transition Detection by Temperature Sensitive Paint at Cryogenic Temperatures in the European Transonic Windtunnel (ETW)," 20th Internatinal Congress on Instrumentation in Aerospace Simulatin Facilities (ICIASF), Goettingen, Germany, 2003.

9 Egami, Y., Fey, U., and Quest, J. "Development of New Two-Component TSP for Cryogenic Testing," 45th AIAA Aerospace Sciences Meeting and Exhibit, Reno, NV, 2007.

10 Germain, E. and Quest, J. "The Development and Application of Optical Measurement Techniques for High Reynolds Number Testing in Cryogenic Environment," 43rd AIAA Aerospace Sciences Meeting and Exhibit, Reno, NV, 2005.

11 Fey, U., Konrath, R., Kirmse, T., Ahlefeldt, T., Kompenhans, J., and Egami, Y. "Advanced Measurement Techniques for High Reynolds Number Testing in Cryogenic Wind Tunnels," 48th AIAA Aerospace Sciences Meeting Including the New Horizons Forum and Aerospace Exposition, Orlando, FL, 2010.

12 Iijima, Y., Egami, Y., Nishizawa, A., Asai, K., Fey, U., and Engler, R. H. "Optimization of TemperatureSensitive Paint Formulation for Large-Scale Cryogenic Wind Tunnels," 20th International Congress on Instrumentation in Aerospace Simulation Facilities (ICIASF), Goettingen, Germany, 2003.

13 Asai, K., Amao, Y., Iijima, Y., Okura, I., and Nishide, H., "Novel Pressure-Sensitive Paint for Cryogenic and Unsteady Wind-Tunnel Testing," Journal of Thermophysics and Heat Transfer, Vol. 16, No. 1, 2002, pp. 109115.

14 Jordan, J. D., Watkins, A. N., Weaver, W. L., Dale, G. A., and Navarra, K. R. "Sol-Gel-Based Pressure-Sensitive Paint Development," 37th AIAA Aerospace Sciences Meeting and Exhibit, Reno, NV, 1999.

15 Watkins, A. N., Goad, W. K., Obara, C. J., Sprinkle, D. R., Campbell, R. L., Carter, M. B., Pendergraft, O. C., Bell, J. H., Ingram, J. L., Oglesby, D. M., Underwood, P. J., and Humber, L. R. "Flow Visualization at Cryogenic Conditions Using a Modified Pressure Sensitive Paint Approach," 43rd AIAA Aerospace Sciences Meeting and Exhibit, Reno, NV, 2005.

16 Watkins, A. N., Leighty, B. D., Lipford, W. E., Oglesby, D. M., Goodman, K. Z., Goad, W. K., Goad, L. R., and Massey, E. A. "The Development and Implementation of a Cryogenic Pressure Sensitive Paint System in the National Transonic Facility," 47th AIAA Aerospace Sciences Meeting including The New Horizons Forum and Aerospace Exposition, Orlando, FL, 2009.

17 Gartenberg, E., Weinstein, L. M., and Lee, E. E., "Aerodynamic Investigation with Focusing Schlieren in a Cryogenic Wind Tunnel," AIAA Journal, Vol. 32, No. 6, 1994, pp. 1242-1249. 
18 Shirinzadeh, B., Herring, G. C., and Barros, T. "Demonstration of Imaging Flow Diagnostics Using Rayleigh Scattering in Langley 0.3-Meter Transonic Cryogenic Tunnel," NASA Technical Note 1999-208970, Hampton, VA, 1999.

19 Quest, J. and Konrath, R. "Accepting a Challenge - The Development of PIV for Application in pressurized cryogenic Wind Tunnels," 41st AIAA Fluid Dynamics Conference and Exhibit, Honolulu, HI, 2011.

20 Konrath, R., Agocs, J., Geisler, R., Otter, D., Roosenboom, E. W. M., Wolf, T., and Quest, J. "Flow Field Measurements by PIV at High Reynolds Numbers," 51st AIAA Aerospace Sciences Meeting including the New Horizons Forum and Aerospace Exposition, Grapevine, TX, 2013.

21 Konrath, R., Geisler, R., Agocs, J., Otter, D., Ehlers, H., Philipp, F., and Quest, J. "High-Speed PIV Applied to Wake of NASA CRM Model in ETW Under High Re-Number Stall Conditions for Sub- and Transonic Speeds," 53rd AIAA Aerospace Sciences Meeting, Kissimmee, FL, 2015.

22 Konrath, R., Geisler, R., Agocs, J., Otter, D., Ehlers, H., Philipp, F., and Quest, J. "Tracking the Nacelle Vortex Above Aircraft Wing in the ETW at Real Mach- and Reynolds Numbers by Means of PIV," 53rd AIAA Aerospace Sciences Meeting, Kissimmee, FL, 2015.

23 Willert, C., Stockhausen, G., Beversdorff, M., Klinner, J., Lempereur, C., Barricau, P., Quest, J., and Jansen, U., "Application of Doppler global velocimetry in cryogenic wind tunnels," Experiments in Fluids, Vol. 39, 2005, pp. 420-430.

24 Michael, J. B., Edwards, M. R., Dogariu, A., and Miles, R. B., "Femtosecond laser electronic excitation tagging for quantitative velocity imaging in air," Applied Optics, Vol. 50, No. 26, 2011, pp. 5158-5162.

25 Danehy, P. M., O'Byrne, S., Frank, A., Houwing, P., Fox, J. S., and Smith, D. R., "Flow-Tagging Velocimetry for Hypersonic Flows Using Fluorescence of Nitric Oxide," AIAA Journal, Vol. 41, No. 2, 2003, pp. $263-271$.

26 Sanchez-Gonzalez, R., Srinivasan, R., Bowersox, R. D. W., and North, S. W., "Simultaneous velocity and temperature measurements in gaseous flow fields using the VENOM technique," Optics Letters, Vol. 36, No. 2, 2011, pp. 196-198.

27 Ribarov, L. A., Wehrmeyer, J. A., Pitz, R. W., and Yetter, R. A., "Hydroxl tagging velocimetry (HTV) in experimental air flow," Applied Phyics B, Vol. 74, 2002, pp. 175-183.

28 Pitz, R. W., Wehrmeyer, J. A., Ribarov, L. A., Oguss, D. A., Batliwala, F., DeBarber, P. A., Deusch, S., and Dimotakis, P. E., "Unseeded molecular flow tagging in cold and hot flows using ozone and hydroxyl tagging velocimetry," Measurement Science and Technology, Vol. 11, 2000, pp. 1259-1271.

29 Sijtesma, N. M., Dam, N. J., Klein-Douwel, R. J. H., and ter Meulen, J. J., "Air Photolysis and Recombination Tracking: A New Molecular Tagging Velocimetry Scheme," AIAA Journal, Vol. 40, No. 6, 2002, pp. 1061-1064.

30 Miles, R. B., Zhou, D., Zhang, B., Lempert, W. R., and She, Z. S., "Fundamental Turbulence Measurements by Relief Flow Tagging," AIAA Journal, Vol. 31, No. 3, 1993, pp. 447-452.

31 Bathel, B. F., Danehy, P. M., Inman, J. A., Jones, S. B., Ivey, C. B., and Goyne, C. P., "Multiple Velocity Profile Measurements in Hypersonic Flows Using Sequentially-Imaged Fluorescence Tagging," AIAA Journal, Vol. 49, No. 9, 2011, pp. 1883-1896.

32 DeLuca, N. J., Miles, R. B., Kulatilaka, W. D., Jiang, N., and Gord, J. R. "Femtosecond Laser Electronic Excitation and Tagging (FLEET) Fundamental Pulse Energy and Spectral Response," 30th AIAA Aerodynamic Measurement Technology and Ground Testing Conference, Atlanta, GA, 2014.

33 Edwards, M. R., Dogariu, A., and Miles, R. B., "Simultaneous Temperature and Velocity Measurements in Air with Femtosecond Laser Tagging," AIAA Journal, 2015, pp. 1-9 (Pre-publish).

34 Michael, J. B., Edwards, M. R., Dogariu, A., and Miles, R. B. "Velocimetry by femtosecond laser electronic excitation and tagging (FLEET) of air and nitrogen," 50th AIAA Aerospaces Sciences Meeting including the New Horizons Forum and Aerospace Exposition, Nashville,TN, 2012.

35 Danehy, P. M., Hires, D. V., Johansen, C. T., Bathel, B. F., Jones, S. B., Gragg, J. G., and Splinter, S. C. "Quantitative Spectral Radiance Measurements in the HYMETS Arc Jet," 50th AIAA Aerospace Sciences Meeting including the New Horizons Forum and Aerospace Exposition, Nashville, TN, 2012.

36 Peters, C. J., Danehy, P. M., Bathel, B. F., Jiang, N., Calvert, N. D., and Miles, R. B. "Precision of FLEET velocimetry using High-Speed CMOS Camera Systems," to be presented at AIAA Aviation 2015, Dallas, TX, 2015.

37 Miles, R. B., Grinstead, J., Kohl, R. H., and Diskin, G., "The RELIEF flow tagging technique and its application 
in engine testing facilities and for helium-air mixing studies," Measurement Science and Technology, Vol. 11, 2000, pp. 1272-1281.

38 Zheng, Q. and Klewicki, J. C., "A fast data reduction algorithm for molecular tagging velocimetry: the decoupled spatial correlation technique," Measurement Science and Technology, Vol. 11, 2000, pp. 1282-1288.

39 Gendrich, C. P. and Koochesfahani, M. M., "A spatial correlation technique for estimating velocity fields using molecular tagging velocimetry (MTV)," Experiments in Fluids, Vol. 22, 1996, pp. 67-77.

40 Danehy, P. M., Bathel, B. F., Calvert, N., Dogariu, A., and Miles, R. P. "Three-component velocity and acceleration measurement using FLEET," 30th AIAA Aerodynamic Measurement Technology and Ground Testing Conference, Atlanta, GA, 2014.

41 Malik, N. A., Dracos, T., and Papantoniou, D. A., "Particle tracking velocimetry in three-dimensional flows; Part II: Particle tracking," Experiments in Fluids, Vol. 15, 1993, pp. 279-294.

$42 \mathrm{Li}$, D., Zhang, Y., Sun, Y., and Yan, W., "A multi-frame particle tracking algorithm robust against input noise," Measurement Science and Technology, Vol. 19, 2008, pp. 1-11.

43 Cierpka, C., Lutke, B., and Kaehler, C. J., "Higher order mult-frame particle tracking velocimetry," Experiments in Fluids, Vol. 54, 2013, pp. 1533-1544.

44 Lempert, W. R., Jiang, N., Sethuram, S., and Samimy, M., "Molecular Tagging Velocimetry Measurements in Supersonic Microjets," AIAA Journal, Vol. 40, No. 6, 2002, pp. 1065-1070.

45 Limbach, C. and Miles, R. B. "Characterization of Dissociation and Gas Heating in Femtosecond Laser Plasma with Planar Rayleigh Scattering and Rayleigh Scatting Polarimetry," 53rd AIAA Aerospace Sciences Meeting, Kissimmee, FL, 2015.

46 Stainback, P. C., Johnson, C. B., and Basnett, C. B. "Preliminary Measurements of Velocity, Density and Total Temperature Fluctuations in Compressible Subsonic Flow," AIAA 21st Aerospace Sciences Meeting and Exhibit, Reno, NV, 1983.

47 Johnson, C. B. and Stainback, P. C. Dynamic Measurement of Total Temperature, Pressure, and Velocity in the Langley 0.3-Meter Transoic Cryogenic Tunnel; NASA Technical Paper 2584: Hampton, VA, 1986.

48 White, F. M. Compressible Boundary-Layer Flow. In Viscous Fluid Flow; McGraw Hill: New York, NY, 2006; pp 505-570.

49 Duan, L., Beekman, I., and Martin, M. P., "Direct numerical simulation of hypersonic turbulent boundary layers. Part 3. Effect of Mach number," Journal of Fluid Mechanics, Vol. 672, 2011, pp. 245-267. 